\title{
Extending Our Reach: Looking to the Future for the Marshall Journal of Medicine
}

\author{
Darshana T. Shah, PhD'
}

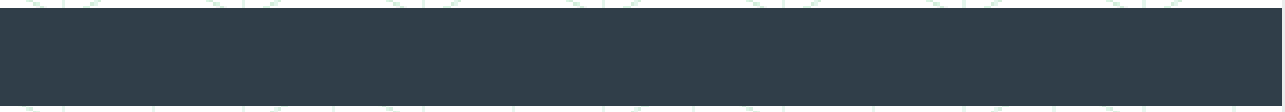

Author affiliations are

listed at the end of this

article.

Correspondence to:

Darshana T. Shah, PhD-

Marshall Unviersity

Joan C. Edwards

School of Medicine

shah@marshall.edu

\section{KEYWORDS}

MJM, PlumX metrics, DOAJ, COPE

The Marshall Journal of Medicine has completed three years of publication. Some notable developments may have been recognized by most of our readers, but others may have passed unnoticed. Therefore, I would like to summarize past and recent developments in the Marshall Journal of Medicine (Journal, MJM).

The new development has been made to measure the impact beyond google scholar and crossref. MJM offers alt metrics such as PlumX to measure the impact of the published papers. Each published paper now shows the PlumX metrics widgets and download counts, which are helpful tools for authors to see their impact. PlumX metrics gives better insight into user engagement with the publication by tracking factors such as several downloads and the mention of the research in news or tweet.

The Journal now offers Native Streaming, a new feature supported by Digital Commons. Native Streaming allows authors to upload multimedia files as the primary file during submission; it includes usage metrics, supports closed-captioning, and provides unlimited storage space. Additionally, readers will be able to view content without having to download it.

MJM also offers the Dashboard to quickly pull up readership for individual articles. Explore recent data, all-time, or a custom date range. The Author Dashboard is a personalized reporting tool for authors with works published in MJM. As an author, you can easily access your dashboard to view upto-date download information for every work you publish, as well as global insights into the sources of readership. You also have options to share your data with others and export statistics for use in external applications.

The Journal is steadily expanding its authorship beyond the mountain state and Appalachia, receiving articles from Nigeria, India, and Libya. Peer review remains a vital component of the assessment of submitted articles. With the help of 500 plus reviewers, MJM follows a rigorous peer-review process. With an acceptance rate of $66.6 \%$, MJM has published a total of 212 peer-reviewed articles since July 2015. The Journal global readership map has expanded and includes 179 countries with 
greater than 43,000 downloads since its inception. The Journal is indexed in Google Scholar, CLOCKSS, Portico, OCLC (WorldCat), ProQuest, Serial Solutions/ Summon, EBSCO Discover, and is a member of the Directory of Open Access Journal (DOAJ) and the Committee on Publication Ethics (COPE).

We hope to have improved the submission process, and the appeal of the Journal cover for our authors and readers. MJM Editorial Board members wish to encourage more contribution from the scientific community to ensure the continued success of the Journal.

\section{AUTHOR AFFILIATIONS}

1. Marshall University Joan C. Edwards School of Medicine, Huntington, West Virginia 